Disponível em

http://www.anpad.org.br/rac

RAC, Rio de Janeiro, v. 20, n. 1, art. 5, pp. 84-105, Jan./Fev. 2016

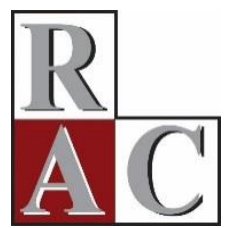

\title{
Sistemas de Reputação: Um Estudo sobre Confiança e Reputação no Comércio Eletrônico Brasileiro
}

\author{
Reputation Systems: A Sudy on Trust and Reputation in Brazilian Electronic \\ Commerce
}

Douglas de Lima Feitosa ${ }^{1}$

Leandro Sumida Garcia ${ }^{2}$

Fundação Getulio Vargas - Escola de Administração de Empresas de São Paulo ${ }^{1}$ Universidade Federal do $\mathrm{ABC}^{2}$

Artigo recebido em 25.07.2013. Última versão recebida em 30.03.2015. Aprovado em 06.04.2015. 


\title{
Resumo
}

Os sistemas de reputação desempenham um papel relevante no comércio eletrônico atual, ao passo que o processo de elaboração e aferição de índices para lojas virtuais aparece como um dos determinantes na decisão de compra do consumidor. Assim, à luz da relação teórica entre os construtos confiança e reputação, este trabalho tem o objetivo de analisar a coerência dos indicadores apresentados por sistemas de reputação de dois grandes sites brasileiros de avaliação de empresas. Foram utilizados dados de 90 empresas do comércio eletrônico brasileiro que possuíam selos de reputação e confiança em seu portfólio. Para tanto, utilizou-se a técnica ANOVA para comparação das médias de reputação de empresas que possuem diferentes selos de confiança. Os resultados demonstram que não existe coerência entre os sistemas de reputação brasileiros e reforçam a existência de outras variáveis preditoras no modelo teórico para avaliação dos escores providos por essas empresas. As conclusões deste trabalho ressaltam a necessidade de realização de novos estudos para compreensão dos vieses e variáveis inerentes aos sistemas de reputação brasileiros, viabilizando o aprofundamento teórico da relação entre os construtos confiança e reputação.

Palavras-chave: sistemas de reputação; comércio eletrônico; confiança; reputação; decisão de compra.

\begin{abstract}
Reputation systems play an important role in the current electronic commerce scenario, as formulation and standardization of indices for web-stores contribute to determine customers' buying decisions. In light of the theoretical relationship between the constructs trust and reputation, this paper intends to analyze the coherence between evaluation indexes built by reputation systems from two big business review sites. We used data from 90 Brazilian e-commerce companies (that maintain .br domains) which have achieved both reputation seals and trust seals. An ANOVA test was used in order to compare the mean of the reputation index of companies that have different trust seals. Results showed no coherence between the reputation systems and also increased the likelihood of existence of other predictive variables in this theoretical model, when it comes to assessing indices provided for those companies. This paper's conclusions highlight the need for new studies which will allow the comprehension of particularities and variables related to reputation systems in Brazil and thus lead to a more accurate understanding of the theoretical relationship between trust and reputation.
\end{abstract}

Key words: reputation systems; electronic commerce; trust; reputation; buying decision. 


\section{Introdução}

Com a expansão do comércio eletrônico nas últimas décadas, foram elevadas também as preocupações inerentes às questões de segurança e privacidade no comércio eletrônico (Albertin \& Moura, 1998; D. J. Kim, Ferrin, \& Rao, 2008; Hartono, Holsapple, Kim, Na, \& Simpson, 2014). O aumento no número de crimes e golpes na Internet, e, consequentemente, da percepção dos riscos nas compras online, faz com que os consumidores se tornem mais cautelosos, buscando informações a respeito do vendedor e levando-as em consideração na decisão de compra (Karahanna, Williams, Polites, Liu, \& Seligman, 2013). A necessidade de se conhecer mais o comerciante antes dessa decisão incentivou a criação de sistemas de reputação, cujos serviços desempenham um papel relevante no contexto do comércio eletrônico atual (Resnick, Zeckhauser, Friedman, \& Kuwabara, 2000; Zhang, Chen, \& Wang, 2004).

Esses sistemas de reputação atuam como mecanismos que visam à redução da assimetria de informações entre consumidores e vendedores, além de atuarem no estabelecimento de selos e escores que atestam o cumprimento de normas e políticas consideradas como necessárias às lojas que atuam no mercado digital (Josang, Ismail, \& Boyd, 2007; Vavilis, Petkovic, \& Zannone, 2014).

A importância desses sistemas para mercados como o brasileiro, em que a confiança não é totalmente difundida nas práticas sociais e o volume de negócios online ainda é relativamente baixo, é maior do que para mercados onde há uma cultura de confiança e alta experiência em negócios virtuais (Casado \& Pelaez, 2014; Standifird, 2001). Consumidores em potencial levam em consideração avaliações e experiências passadas no momento de decidir por uma determinada loja ou produto (Mudambi \& Schuff, 2010).

No entanto, há diferentes stakeholders no contexto dos sistemas de reputação, incluindo consumidores, vendedores e as empresas que administram os referidos sistemas. Isso conduz ao entendimento de que existem diferentes interesses relacionados à criação e à manutenção dos sistemas de reputação, o que pode incorrer em vieses nos resultados e escores publicados por essas entidades (Wu, Cheng, \& Yen, 2014).

Paralelamente, a literatura nacional sobre sistemas de reputação praticamente inexiste, gerando uma lacuna que diz respeito ao posicionamento desses sistemas perante o comércio eletrônico brasileiro e suas particularidades. Em pesquisa feita em dezembro de 2014, na ferramenta de busca de artigos acadêmicos Web of Knowledge, apenas 13 artigos de autores brasileiros foram encontrados com a tag sistemas de reputação, sendo que nenhum desses se refere à publicação realizada em evento ou periódico nacional. Todavia, em pesquisa realizada pela Fundação de Proteção e Defesa do Consumidor (PROCON, 2013) - órgão de proteção ao consumidor do Estado de São Paulo, é possível identificar varejistas com altos escores de confiança em sistemas de reputação figurando entre as empresas de comércio eletrônico que mais recebem reclamações fundamentadas, por exemplo.

Assim, este trabalho se baseia na relação teórica entre as variáveis confiança e reputação, e nos dados divulgados de dois dos principais sistemas de reputação brasileiros para responder à seguinte questão: Existe coerência entre os escores apresentados pelos sistemas de reputação brasileiros?

No sentido de verificar a relação teórica existente entre os construtos reputação e confiança, no contexto brasileiro, foram coletados dados de lojas virtuais avaliadas pelos sistemas de reputação eBIT e Reclame Aqui. Por meio de uma abordagem quantitativa, foram comparados os índices de reputação de empresas aferidas com diferentes selos de confiança.

É proposta uma abordagem para análise de coerência de selos e escores aferidos por diferentes empresas que atuam no contexto do comércio eletrônico de uma mesma região.

Após esta seção introdutória, este trabalho segue a seguinte estrutura: na próxima seção, são apresentados os principais fundamentos teóricos referentes à temática abordada; em seguida, os 
procedimentos, métricas e métodos utilizados neste trabalho são descritos; na sequência, são apresentados os resultados obtidos; na seção seguinte, são apresentadas as análises dos resultados e as limitações do estudo; e, por fim, são listadas as conclusões e as possibilidades de novos estudos sobre o fenômeno investigado.

\section{Fundamentação Teórica}

As próximas subseções trazem aspectos relacionados à confiança no comércio eletrônico, às questões que influenciam a reputação das empresas perante os consumidores, e ao processo de aferição de selos e escores, por parte de empresas especializadas.

\section{Aspectos da confiança no comércio eletrônico}

O comércio eletrônico é definido como qualquer transação econômica em que compradores e vendedores se comunicam por meio de mídias eletrônicas da Internet, firmam um acordo contratual no que diz respeito ao estabelecimento de preços e entrega de bens/serviços específicos, e consolidam a transação por meio da execução do pagamento e da entrega desses bens/serviços, conforme estabelecido em contrato (Guay \& Ettwein, 1998; Turban \& King, 2004).

Com o aumento do volume de transações realizadas, aumentaram também os eventuais problemas inerentes a esse contexto (D. J. Kim et al., 2008; Hartono et al., 2014). O fato de que, geralmente, o consumidor realiza o pagamento sem o contato direto com os bens ou serviços ofertados representa uma situação oportunista para golpes, resultando em um aumento do risco percebido por parte de quem realiza a compra (Cernev \& Leite, 2005; Kollock, 1999; Kovacs \& Farias, 2004). Outra preocupação em comum está relacionada às práticas de vendas de informações pessoais de clientes por parte de algumas empresas (Liao, Liu, \& Chen, 2011). Desta forma, empresas, acadêmicos e consumidores passaram a destinar uma maior parte de sua atenção às questões que influenciam as percepções de segurança e privacidade neste âmbito (Pavlou, Liang, \& Xue, 2007).

Diversas pesquisas investem na criação de esquemas e mecanismos que facilitem a identificação dos fatores que levam clientes a adquirir confiança em relação a uma determinada empresa, no âmbito do comércio eletrônico (McKnight, Choudhury, \& Kacmar, 2002). De fato, a própria definição de confiança apresenta versões variadas, que estão relacionadas a diferentes ontologias (Butler, 1991; Rempel, Holmes, \& Zanna, 1985; Rotter, 1971): do ponto de vista da psicologia, confiança é definida como uma tendência de confiar em outros indivíduos; do ponto de vista da psicologia social, confiança é definida como uma cognição acerca da entidade em questão; já do ponto de vista da sociologia, confiança é entendida como uma característica do ambiente institucional. Por conta das diferentes caracterizações de confiança, outras pesquisas passaram a abordar o conceito como um fator multivariado, que sofre influência de diversos aspectos, conforme ilustrado no levantamento realizado por McKnight, Choudhury e Kacmar (2002), Tabela 1.

Tabela 1

Aspectos Utilizados para Mensuração do Fator Confiança

\begin{tabular}{llll}
\hline Fatores & Variáveis & Frequência & Frequência Relativa \\
\hline Competência & Competência & 11 & $13 \%$ \\
& Habilidade & 3 & $3 \%$ \\
& Dinamismo & 3 & $3 \%$ \\
\hline
\end{tabular}


Tabela 1 (continuação)

\begin{tabular}{llll}
\hline Fatores & Variáveis & Frequência & Frequência Relativa \\
\hline Benevolência & Disposição & 10 & $12 \%$ \\
& Benevolência & 16 & $19 \%$ \\
& Responsividade & 4 & $5 \%$ \\
\hline Integridade & Integridade & 8 & $9 \%$ \\
& Moralidade & 6 & $7 \%$ \\
& Credibilidade & 1 & $1 \%$ \\
& Confiabilidade & 7 & $8 \%$ \\
& (Reliability) & & $6 \%$ \\
& Confiabilidade & 5 & $7 \%$ \\
(Dependability) & & $4 \%$ \\
& Predição & 6 & $2 \%$ \\
& Abertura & 3 & $1 \%$ \\
\hline Total & Responsabilidade & 2 & $\mathbf{1 0 0 \%}$ \\
\hline
\end{tabular}

Nota. Adaptado de McKnight, D. H., Choudhury, V., \& Kacmar, C. (2002). Developing and validating trust measures for ecommerce: an integrative typology (p. 338). Information Systems Research, 13(3), 334-359. doi: 10.1287/isre.13.3.334.81

Trabalhos recentes abordaram outros aspectos relacionados à confiança. Algumas pesquisas colocaram a qualidade como um fator que aumenta a confiança em empresas do varejo eletrônico (Gregg \& Walczak, 2010; Vieira, 2010; Vieira \& Slongo, 2008). Ballester e Espalhardo (2008) realizaram um experimento com 265 consumidores online e verificaram que a similaridade percebida e o grau de associação entre marcas contribuem para construção da confiança. C. Kim, Tao, Shin e Kim (2010) aplicaram uma Web survey para consumidores coreanos e verificaram que os aspectos técnicos e as declarações de segurança influenciam a percepção de confiança dos consumidores. Outros fatores levantados estão relacionados à justiça no relacionamento com os consumidores; experiência prévia e familiaridade; usabilidade, segurança e privacidade percebidas; e censura de feedbacks negativos (Bae \& Lee, 2011; Chiu, Huang, \& Yen, 2010; Eid, 2011; Pizzutti \& Fernandes, 2010).

Esse interesse pela descoberta dos aspectos de confiança pode ser explicado pela intenção, por parte das empresas, de levar os consumidores a superarem percepções de incerteza e risco, facilitando a realização de transações comerciais (Cho, 2010). Nesse cenário, talvez o maior desafio seja o estabelecimento da confiança inicial, em que o consumidor não possua nenhuma experiência de compra com a empresa e a empresa não possua informações sobre o consumidor (McKnight, Cummings, \& Chervany, 1998).

Duas vertentes da literatura abordam a forma como se dá a geração da confiança inicial: confiança baseada no conhecimento; e confiança baseada na cognição. A literatura relacionada à confiança baseada em cognição explica que o estabelecimento da confiança inicial pode se dar rapidamente em virtude de categorizações sociais, reputação, imagem percebida, disposição, regras e estruturas institucionais, e postura (Benedicktus, Brady, Darke, \& Voorhees, 2010; McKnight et al., 1998; Meyerson, Weick, \& Kramer, 1996). Já a literatura inerente à confiança baseada em conhecimento defende que a confiança é construída a partir das experiências de trocas no contexto social (Lewicki \& Bunker, 1995; Shapiro, Sheppard, \& Cheraskin, 1992). Para este trabalho, considera-se a vertente de confiança baseada na cognição, tendo a reputação como um antecedente à confiança. Segundo H. W. Kim, Xu e Gupta (2012), essa confiança é o principal fator considerado pelos consumidores durante a 
realização da primeira compra junto a um vendedor online, sugerindo que vendedores mais confiáveis possuem uma maior capacidade para captação de novos clientes.

Ainda neste contexto, percebe-se o surgimento de alguns mecanismos de governança, que foram desenvolvidos no sentido de garantir um comportamento confiável por parte das empresas, e elevar a percepção de confiança por parte dos consumidores no comércio eletrônico. Sistemas foram desenvolvidos para atrasar o pagamento até o recebimento de produtos, mediante custo adicional (Smith \& Shao, 2007). Empresas desenvolveram políticas que garantem a devolução do dinheiro do consumidor, até certo montante. Outro mecanismo de governança utilizado é a reputação, uma vez que, apesar de não estar diretamente ligada à obrigatoriedade da execução do contrato firmado entre comprador e vendedor, não incorre em custos adicionais.

\section{Reputação no comércio eletrônico}

Reputação pode ser definida como uma medida de quão desejável é uma entidade, conforme esse valor seja estabelecido por uma pessoa ou grupo de pessoas externas à entidade em questão (Standifird, Weinstein, \& Meyer, 1999; Wartick, 2002). Apesar de tentarem realizar a manutenção de suas respectivas reputações junto aos consumidores, as empresas (entidades) estão sujeitas às avaliações de empresas externas a ela.

Uma reputação alta/forte pode ser vista como garantia contra comportamentos abusivos de vendedores (Standifird, 2001; You, Liu, Xia, \& Lv, 2011). Tendo em vista que o preço total de um produto é a combinação de um preço de compra, custos de busca do produto e custos de desapontamento na compra, uma boa reputação pode ser utilizada para minimizar esses custos de desapontamento na compra (H. W. Kim \& Xu, 2007). A reputação pode ainda criar barreiras de entrada em um mercado digital, uma vez que novos entrantes podem considerar inviável a competição com vendedores já consolidados no âmbito em questão (Melnik \& Alm, 2002). Outros estudos ainda sugerem que vendedores com alta reputação possuem uma maior taxa de crescimento, se comparados aos vendedores com menores índices de reputação (Lin, Li, Jamananchi, \& Huang, 2006).

Visando identificar os reais impactos da reputação, diversas pesquisas apresentaram métricas no contexto do comércio eletrônico. Standifird (2001), por exemplo, realizou um estudo que demonstrou a existência de impactos das reputações positivas e negativas nos valores obtidos por vendedores do site eBay.com. Ba e Pavlou (2002) também fizeram experimentos no contexto do site eBay.com e verificaram que a reputação de um vendedor, em seus registros de feedback, tem um importante papel na intenção de compra de consumidores, ao passo que lhes dá confiança para realização de lances com maior valor. Corbitt, Thanasankit e Yi (2003) aplicaram uma Web survey para usuários de Internet na Nova Zelândia, para identificar os fatores que levam à confiança por parte de consumidores no comércio eletrônico, e identificaram que a confiança é afetada predominantemente por três fontes: reputação do comércio eletrônico; consumidores; e web site da empresa.

Já Amblee e Bui (2008) realizaram uma análise de 395 e-books ofertados no site Amazon.com e verificaram que aqueles com melhores avaliações iniciais potencializavam os feedbacks de consumidores. Outras pesquisas ratificam o papel da reputação como um dos antecedentes da confiança no comércio eletrônico (Casaló, Flavián, \& Guinalíu, 2011; Edelman, 2011; Saastamoinen, 2009).

Essa lógica de mercado, à qual estão sujeitas as empresas e os consumidores do comércio eletrônico, diz respeito à limitação da habilidade das empresas no sentido de manipular sua própria reputação, que fica sujeita à intenção de entidades avaliadoras, no que concerne à inclusão dessas tentativas de influências das empresas em sua avaliação (Standifird, 2001). Yoo e Kim (2012) verificaram que os sistemas de reputação podem ser utilizados para potencializar questões de popularidade, política de preços e posicionamento no mercado. Assim, torna-se crucial para o decorrer de seus negócios que as empresas sejam aferidas com altos índices de reputação. 


\section{Sistemas de reputação}

A reputação pode ser ainda mais crítica em um ambiente online, se este for comparado com o ambiente de negócios tradicional. Se a empresa realiza negócios exclusivamente em ambiente digital, os consumidores geralmente buscam por espaços para discussão/feedback nos websites das empresas com as quais pretendem negociar ou por empresas provedoras de informação, no sentido de estabelecer a confiança inicial em relação aos vendedores (Fuller, Serva, \& Benamati, 2007; Utz, Matzat, \& Snijders, 2009). Esses mecanismos que englobam feedbacks e escores acerca de lojas virtuais são conhecidos como sistemas de reputação (Casaló et al., 2011). Em nível mundial, há websites que utilizam esses sistemas, como BBBOnline.org, TRUSTe.com, Epinions.com, NextTag.com e BizRate.com. No Brasil, os sistemas de reputação mais conhecidos são eBit.com.br e ReclameAqui.com.br.

Sistemas de reputação agregam informações de consumidores acerca das experiências obtidas, por meio das transações com empresas. As informações representadas nesses sites são compreendidas como um mecanismo para construção da confiança dos consumidores (Swamynathan, Almeroth, \& Zhao, 2010). Em alguns casos, esses sistemas também atuam na verificação de determinados padrões ou requerimentos considerados importantes para redução dos riscos percebidos por parte dos consumidores, por meio da aferição de selos e certificados para as empresas inseridas em seu portfólio (Edelman, 2011; Park, Bhatnagar, \& Rao, 2010).

Como o estabelecimento de confiança está relacionado ao processo decisório de consumo, a compreensão desses sistemas de reputação tem se tornado objeto de interesse por parte de empresas e pesquisadores (Fuller et al., 2007). Ao prover informações que reduzem a incerteza na percepção dos consumidores, os sistemas de reputação fomentam a criação de confiança ao fornecer percepções de diversos consumidores que avaliam empresas de acordo com o que consideram como comportamento apropriado (Ba \& Pavlou, 2002).

Os sistemas de reputação visam, em geral, à minimização do problema de assimetria de informação entre as entidades (compradores e vendedores) que participam do processo de comércio eletrônico, e encorajam um comportamento que induz à confiança (Benlian \& Hess, 2011). A participação em um sistema de reputação normalmente sinaliza que a empresa é confiável e pode representar um incentivo para o cumprimento de obrigações contratuais inerentes a uma transação. Nesse ambiente, também são percebidos incentivos para que sejam dados feedbacks confiáveis (Saastamoinen, 2009).

A ideia básica no que concerne aos sistemas de reputação envolve a avaliação dos atores envolvidos no processo de negociação, geralmente ocorrendo após o final da transação, e o uso de avaliações agregadas para gerar uma medida de confiança ou reputação (Josang et al., 2007). Assim, dependendo dos escores gerados, um sistema de reputação pode apresentar medidas de confiança ou reputação, o que leva à seguinte hipótese a ser testada nesse trabalho:

$\mathbf{H}_{1}$ : Dada a relação teórica entre os construtos confiança e reputação, empresas que apresentam altos escores de reputação também apresentam altos escores de confiança.

A seção a seguir apresenta os aspectos metodológicos desta pesquisa.

\section{Metodologia}

Esta pesquisa propõe uma análise da coerência de escores de confiança e reputação disponibilizados por sistemas de reputação de duas empresas do comércio eletrônico brasileiro, por meio de uma abordagem quantitativa (Black, 1999). 
Foram utilizados os dados secundários de escores de dois sistemas de reputação: eBIT, cujos escores foram interpretados como medidas do construto confiança; e Reclame Aqui, cujos escores foram interpretados como medidas do construto reputação. Tais informações foram coletadas na primeira quinzena do mês de novembro de 2012.

Dentre as vantagens da utilização de dados secundários para representação de construtos, podem ser mencionadas (Houston, 2004): representam decisões reais, feitas por tomadores de decisão reais, em ambientes reais; dados secundários são menos vulneráveis aos vieses de respondentes; e facilidade de acesso e coleta.

Ressalta-se que o uso de escores de sistemas de reputação para composição dos construtos citados anteriormente é uma prática efetuada em outros estudos (Azderska \& Jerman-Blazic, 2013; Whitmeyer, 2000; Zhou \& Hwang, 2007).

As subseções a seguir descreverão os critérios e os procedimentos utilizados nos processos de operacionalização de construtos, coleta e tratamentos dos dados.

\section{Operacionalização dos construtos e coleta de dados}

Abaixo, são descritos os critérios utilizados por ambos os sistemas de reputação utilizados como referência nesse estudo.

\section{Sistema de reputação eBIT (construto confiança)}

O sistema de reputação eBIT faz parte da e-bit Informação, que é uma empresa com informações inerentes ao comércio eletrônico, sendo uma das principais fontes utilizadas por consumidores brasileiros que possuem intenção de realizar compras em lojas virtuais de diferentes segmentos do mercado. Para manutenção do sistema, é cobrada uma taxa de afiliação para que empresas sejam avaliadas, recebam selos que atestam confiança na compra online, recebam orientações para captação de novos clientes, e participem do portfólio da eBIT. Informações do site indicam que o sistema de reputação da eBIT possui mais de 2.200 lojas virtuais conveniadas (Ebit A Certificação dos Consumidores, n.d.a).

Qualquer consumidor pode acessar o site do sistema eBIT e verificar todas as lojas virtuais conveniadas de acordo com suas características, incluindo: selo obtido, segmento do mercado, formas aceitas de pagamento, política de devolução adotada, abrangência geográfica para entrega, e formas utilizadas para entrega dos produtos/serviços.

No sistema de reputação da eBIT, as avaliações dos consumidores são obtidas por meio de pesquisas de satisfação de compra e de pós-venda das lojas conveniadas. Cada consumidor avalia as empresas em dez diferentes critérios, que podem variar de acordo com o teor dos produtos/serviços vendidos. Cada categoria é medida por um item de questionário, que utiliza uma escala tipo Likert de cinco pontos.

Ao final de cada ciclo de avaliação, são calculadas as médias das avaliações de todos os consumidores para cada item, que são convertidas em medalhas, respeitando os seguintes critérios: critérios com média $x \leq 3$ recebem medalha Bronze; critérios com média $3<x \leq 4$ recebem medalha Prata; critérios com média $4<x \leq 5$ recebem medalha Ouro.

Os selos consolidados que são aferidos às lojas virtuais do sistema de reputação da eBIT são entregues conforme o atendimento de alguns critérios, incluindo a média geral das avaliações realizadas por consumidores. A Tabela 2 apresenta os critérios utilizados para entrega de selos. 
Tabela 2

Critérios para Aferição de Selos para Lojas Virtuais Conveniadas ao Sistema de Avaliação eBIT

\begin{tabular}{|c|c|c|}
\hline ebit Bronze & ebit Prata & ebit Ouro \\
\hline $\begin{array}{l}\text { Para alcançar a medalha } \\
\text { BRONZE, é preciso } \\
\text { atingir os seguintes } \\
\text { quesitos todos os dias, } \\
\text { pelos últimos } 90 \text { dias: }\end{array}$ & $\begin{array}{l}\text { Para alcançar a medalha } \\
\text { PRATA, é preciso atingir } \\
\text { os seguintes quesitos } \\
\text { todos os dias, pelos } \\
\text { últimos } 90 \text { dias: }\end{array}$ & $\begin{array}{l}\text { Para alcançar a medalha } \\
\text { OURO, é preciso atingir } \\
\text { os seguintes quesitos } \\
\text { todos os dias, pelos } \\
\text { últimos } 90 \text { dias: }\end{array}$ \\
\hline $\begin{array}{l}\text { Ter, no mínimo, } 6 \\
\text { pesquisas de avaliação no } \\
\text { ato da compra; }\end{array}$ & $\begin{array}{l}\text { Ter, no mínimo, } 15 \\
\text { pesquisas de avaliação no } \\
\text { ato da compra; }\end{array}$ & $\begin{array}{l}\text { Ter, no mínimo, } 50 \\
\text { pesquisas de avaliação no } \\
\text { ato da compra. }\end{array}$ \\
\hline $\begin{array}{l}\text { Ter, no mínimo, } 2 \\
\text { pesquisas referentes a } \\
\text { serviços de entrega; }\end{array}$ & $\begin{array}{l}\text { Ter, no mínimo, } 5 \\
\text { pesquisas referentes a } \\
\text { serviços de entrega; }\end{array}$ & $\begin{array}{l}\text { Ter, no mínimo, } 15 \\
\text { pesquisas referentes a } \\
\text { serviços de entrega. }\end{array}$ \\
\hline $\begin{array}{l}\text { Ter nota geral dada pelos } \\
\text { seus clientes menor do } \\
\text { que } 3^{*} \text {. }\end{array}$ & $\begin{array}{l}\text { Nota geral dada pelos seus } \\
\text { clientes igual ou superior } \\
\text { a } 3^{*} \text {. }\end{array}$ & $\begin{array}{l}\text { Nota geral dada pelos seus } \\
\text { clientes igual ou superior } \\
\text { a } 4 * \text {. }\end{array}$ \\
\hline
\end{tabular}

ebit Diamante

Para alcançar a medalha

DIAMANTE, é preciso

atingir os seguintes

quesitos todos os dias,

pelos últimos 90 dias:

Ter, no mínimo, 1000

pesquisas de avaliação no ato da compra;

Ter, no mínimo, 20

pesquisas referentes a serviços de entrega;

Ter menos de $15 \%$ de atraso na entrega;

Obter índice igual ou superior a $85 \%$ em probabilidade de consumidores voltarem a comprar na loja;

Trabalhar com protocolo de segurança SSL;

Garantir sigilo no tratamento de informações de clientes;
Ativa na e-bit há pelo menos 90 dias;

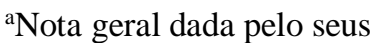 clientes igual ou superior a 4.

Nota. ${ }^{a}$ Média das médias das categorias avaliadas pelos consumidores. Fonte: Adaptado de Ebit A Certificação dos Consumidores. (n.d.a). Conheça as medalhas E-bit. Recuperado de http://www.ebit.com.br/medalhas-e-bit

Inicialmente, buscou-se identificar lojas virtuais conveniadas e avaliadas pelo sistema de reputação eBIT que também possuíssem índices/avaliações no sistema de reputação do site Reclame Aqui para composição de uma amostra que viabilizasse a realização dos testes para análise da coerência entre os sistemas de reputação. Como ponto de início, foi utilizada a lista das 100 empresas ${ }^{(1)}$ eleitas como as mais queridas do sistema eBIT, em 2011. Essa lista é composta a partir do voto popular dos consumidores, anualmente, e faz parte dos resultados do Prêmio E-bit Melhores lojas do E-commerce (Ebit Empresa, n.d.). Nela, constam a posição, o nome da loja e o selo eBIT das empresas.

Foram coletados dados secundários que representam as seguintes informações: nome da loja e selo obtido. 


\section{Sistema de reputação reclame aqui (construto reputação)}

O site www.reclameaqui.com.br (recuperado em 05, novembro, 2012) disponibiliza um sistema de reputação que é baseado em reclamações realizadas por clientes que tiveram qualquer tipo de problema em alguma das etapas do processo de compra (Ex.: pré-compra, compra, pós-compra). O sistema é aberto a qualquer usuário de Internet que preencha corretamente o cadastro no site. Os consumidores podem realizar reclamações que são publicadas no site e um aviso é encaminhado à empresa reclamada, caso possua seu Serviço de Atendimento ao Cliente cadastrado no Reclame Aqui.

Neste sistema, as empresas poderão responder a qualquer momento, publicando, assim, a resposta à reclamação do consumidor, desde que estejam cadastradas no site. As reclamações cadastradas no Reclame Aqui gerarão um ranking atualizado das empresas, conforme critérios de número de reclamações, tempo de resposta, ausência de resposta, índice de solução, número de avaliações, nota do reclamante e índice de voltar a fazer negócio com a empresa, considerados a partir do momento da publicação e das respostas das mesmas. A empresa declara que o sistema de reputação é totalmente automatizado, não havendo interferência de operador na geração dos dados de ranking.

No sistema de reputação do ReclameAqui (n.d.a), são analisados quatro critérios para determinar uma nota de 0 (zero) a 10 (dez) para cada empresa. Os critérios possuem pesos distintos, conforme abaixo:

. Índice de Resposta às Reclamações (IR) - 20\%;

. Média das Avaliações dos Consumidores (MA) - 30\%;

. Índice de Solução de Problemas (IS) - 30\%;

- Índice de Novos Negócios (Voltaria a fazer negócios?) (IN) - 20\%.

Antes de calcular os índices de reputação de uma empresa, há três condições que precisam ser satisfeitas:

A empresa deve possuir mais de $10(\mathrm{dez})$ reclamações. Se possuir um índice de resposta inferior a $50 \%$, ela automaticamente é categorizada como NẪO RECOMENDADA. Isso é feito para garantir que empresas que não respondem a seus consumidores não sejam beneficiadas, pois todos os parâmetros analisados são obtidos a partir do atendimento realizado pelas empresas.

- A empresa deve possuir um número de avaliações igual ou superior a 10 (dez). Se possuir menos, a ela será atribuído o conceito SEM ÍNDICE.

- A empresa deve possuir tempo de cadastro junto ao site superior a 30 (trinta) dias. Se possuir menos, a ela será atribuído o conceito SEM ÍNDICE ${ }^{(2)}$.

Tendo as condições acima atendidas, o cálculo da Avaliação do RA (AR) é realizado por meio de uma média ponderada baseada nos critérios já citados. Todos esses possuem valores que variam de 0 (zero) a 100 (cem), com exceção da Média das Avaliações dos Consumidores (MA) (varia de 0 a 10). Assim, o valor da média das avaliações é multiplicado por 10, para que seja colocada na mesma escala dos demais critérios. Desta maneira, a fórmula para obtenção da Avaliação do RA é a seguinte:

$$
\mathrm{AR}=((\mathrm{IR} * 2)+(\mathrm{MA} * 10 * 3)+(\mathrm{IS} * 3)+(\mathrm{IN} * 2)) / 100
$$

Em que:

. Para $8 \leq \mathrm{AR} \leq 10$, Reputação = ÓTIMO

. Para $7 \leq \mathrm{AR} \leq 7.9$, Reputação $=\mathrm{BOM}$

- Para $6 \leq \mathrm{AR} \leq 6.9$, Reputação = RAZOÁVEL 
- Para $5 \leq \mathrm{AR} \leq 5.9$, Reputação $=$ RUIM

- Para AR < 5, Reputação = NÃO RECOMENDADA

A coleta dos índices de reputação deu-se em paralelo à coleta dos índices de confiança. Para tanto, utilizou-se a ferramenta de busca do site do Reclame Aqui. Neste contexto, foram coletados dados secundários que estão relacionados à reputação de 90 empresas do comércio eletrônico brasileiro. Mais especificamente, foram coletados os escores referentes à Média das Avaliações dos Consumidores (MA) e à reputação consolidada, uma vez que o foco para análise de coerência dos sistemas de reputação é a comparação dos índices de confiança e reputação. A Tabela 3 apresenta os dados coletados para as 90 lojas virtuais que compõem a amostra deste trabalho.

Tabela 3

\section{Dados das Empresas que Compõem a Amostra do Estudo}

\begin{tabular}{lll}
\hline Lojas & Selo ebit (Consolidado) & Nota do Consumidor (RA) \\
\hline Pão de Açúcar & Ouro & 5,67 \\
Lojas Insinuante & Ouro & 5,5 \\
Sèpha Perfumaria & Diamante & 5,93 \\
Apetrexo.com & Prata & 1,78 \\
AutoZ & Ouro & 5,38 \\
Bamboo & Bronze & 5,55 \\
Livraria da Travessa & Diamante & 5,8 \\
Tico Tica Bum & Prata & 5,38 \\
Farma Delivery & Ouro & 6,57 \\
Droga Raia & Diamante & 5,25 \\
Athletic & Prata & 5,49 \\
Cirúrgica Passos & Ouro & 4,63 \\
Fujioka & Bronze & 5,53 \\
Clovis Calçados & Prata & 6,5 \\
Hering & Ouro & 5,24 \\
Renner & Ouro & 5,23 \\
Tiguana & Ouro & 4,68 \\
Safari Shop & Prata & 5,97 \\
Lojas KD & Prata & 4,39 \\
Megamamute & Ouro & 5,24 \\
Kmdevantagens & Ouro & 3,4 \\
Mania Virtual & Ouro & 4,54 \\
Editora Abril & Ouro & 5,54 \\
Lojas Colombo & Diamante & 4,17 \\
Zelo & Diamante & 6,86 \\
Olympus & Bronze & 6,09 \\
\hline & & \\
& & \\
\hline
\end{tabular}


Tabela 3 (continuação)

\begin{tabular}{|c|c|c|}
\hline Lojas & Selo ebit (Consolidado) & Nota do Consumidor (RA) \\
\hline Siciliano & Diamante & 6,05 \\
\hline Eletro Shopping & Ouro & 3,81 \\
\hline Casa \& Video & Ouro & 5,56 \\
\hline Camisaria Colombo & Prata & 4,76 \\
\hline Pipper Joias & Prata & 4,37 \\
\hline Beleza na Web & Diamante & 7,3 \\
\hline Brastemp & Ouro & 4,29 \\
\hline Videolar.com & Diamante & 4,72 \\
\hline Polishop & Ouro & 5,81 \\
\hline Loja do Flamengo & Prata & 4,38 \\
\hline Lojas MM & Ouro & 3,65 \\
\hline $\mathrm{TAM}^{\mathrm{b}}$ & Prata & 3,53 \\
\hline Fnac & Diamante & 3,74 \\
\hline Onofre em Casa & Diamante & 4,56 \\
\hline eFácil & Ouro & 3,98 \\
\hline Dafiti & Diamante & 6,1 \\
\hline O Boticário & Ouro & 6,39 \\
\hline Centauro & Ouro & 4,74 \\
\hline Sephora (Sacks) & Diamante & 5,82 \\
\hline Posthaus & Diamante & 6,98 \\
\hline Livraria Cultura & Diamante & 6,69 \\
\hline Brandsclub & Prata & 4,92 \\
\hline Aprova Concursos ${ }^{\mathrm{b}}$ & Ouro & 7,89 \\
\hline Passarela Calçados & Diamante & 8 \\
\hline Balão da Informática & Diamante & 5,45 \\
\hline Marisa & Ouro & 6 \\
\hline Kabum! & Diamante & 4,48 \\
\hline $\mathrm{CVC}$ & Ouro & 4,57 \\
\hline Carrefour & Diamante & 3,63 \\
\hline Kalunga & Ouro & 4,86 \\
\hline Compra Fácil & Ouro & 4,63 \\
\hline Ricardo Eletro & Ouro & 3,37 \\
\hline Ultrafarma & Diamante & 4,7 \\
\hline Fast Shop & Diamante & 5,11 \\
\hline Saraiva.com.br & Diamante & 6,27 \\
\hline Walmart & Diamante & 4,81 \\
\hline
\end{tabular}


Tabela 3 (continuação)

\begin{tabular}{|c|c|c|}
\hline Lojas & Selo ebit (Consolidado) & Nota do Consumidor (RA) \\
\hline Extra.com.br & Diamante & 4,93 \\
\hline Pontofrio & Diamante & 5 \\
\hline Magazine Luiza & Diamante & 4,22 \\
\hline Ri Happy & Ouro & 6,47 \\
\hline Toymania & Ouro & 4,6 \\
\hline Bebê Store & Prata & 5,83 \\
\hline Livraria Cultura & Diamante & 6,69 \\
\hline DVD World & Ouro & 4,73 \\
\hline Som Livre & Ouro & 4,92 \\
\hline Panvel Farmácias & Diamante & 6,81 \\
\hline Editora Globo & Prata & 6,83 \\
\hline Arte Pau Brasil & Prata & 4,45 \\
\hline SBS Livraria Virtual & Prata & 6,63 \\
\hline Vida e Cor & Diamante & 8,21 \\
\hline Westwing Home and Living & Prata & 5,4 \\
\hline Lojas Certel & Prata & 8,18 \\
\hline Corpo Perfeito & Diamante & 3,99 \\
\hline Beleza na Web & Diamante & 7,3 \\
\hline Cirúrgica Express & Prata & 2,9 \\
\hline Pedala & Prata & 7,93 \\
\hline Nova Flor & Prata & 3,82 \\
\hline Ingresso Certo ${ }^{b}$ & Bronze & 7,83 \\
\hline Elsevier Editora & Prata & 3,17 \\
\hline Drogasil & Prata & 5,94 \\
\hline Nossa Vitrine & Prata & 4,53 \\
\hline Imperdível & Prata & 5,86 \\
\hline Clube do Desconto & Bronze & 3,85 \\
\hline Qpechincha & Bronze & 3,73 \\
\hline
\end{tabular}

Nota. Fontes: Adaptado de Ebit A Certificação dos Consumidores. (n.d.b). Reputação das lojas. Recuperado de http://www.ebit.com.br/avaliacao-lojas e ReclameAqui. (n.d.b). Rankings das empresas mais reclamadas por categoria. Recuperado de http://www.reclameaqui.com.br/

aLoja recém excluída do portfólio ebit. ${ }^{b}$ Lojas avaliadas por critérios diferenciados.

\section{Tratamento dos dados}

Uma vez coletados os dados das 90 lojas virtuais, iniciou-se o processo de análise dos dados por meio da técnica para comparação de médias conhecida como ANOVA ou Análise de Variância (Anderson, Sweeney, \& Williams, 2011), uma das técnicas mais difundidas academicamente quando se trata de comparação de médias de três ou mais grupos. 
A técnica ANOVA foi utilizada para verificação da coerência entre os sistemas de reputação estudados. Como já mencionado anteriormente, diversos estudos citam a existência de uma relação entre a reputação e o estabelecimento de confiança, no contexto do comércio eletrônico, além de outros fatores mencionados. Neste caso, pretende-se testar a hipótese apresentada na seção anterior, de que empresas com altos escores de confiança possuirão altos escores de reputação, assim como se espera que empresas com baixos escores de confiança possuam baixos escores de reputação.

Desta forma, ao comparar as médias de grupos formados por empresas com selo eBIT Prata + Bronze (Grupo 1), selo eBIT Ouro (Grupo 2) e eBIT Diamante (Grupo 3), espera-se que sejam encontradas diferenças significativas entre as médias dos grupos. Logo, por meio da técnica ANOVA, será operacionalizado o seguinte teste:

$$
\mathbf{H}_{\mathbf{0}}: \mu_{\text {Grupo } 1}=\mu_{\text {Grupo } 2}=\mu_{\text {Grupo } 3}
$$

$\mathbf{H}_{\mathrm{A}}$ : Nem todas as médias são iguais (ao menos uma das médias é diferente das demais)

A priori, foi necessário definir quais escores deveriam ser utilizados na análise. Do ponto de vista dos escores de confiança (eBIT), optou-se pela utilização dos selos aferidos para as empresas, uma vez que não era possível estimar, com segurança (médias dos critérios dadas em medalhas), quais eram as notas dadas para cada critério pelos consumidores. Outro ponto observado é que, independente do selo que a empresa possuísse (Bronze, Prata, Ouro ou Diamante), as notas dadas aos critérios individuais eram igualmente altas, com algumas exceções. Observam-se casos, inclusive, em que empresas com selos mais altos (Diamante, Ouro) possuem médias de avaliações dos consumidores menores do que outras empresas com selos menos representativos (Prata, Bronze). No que concerne aos escores de reputação (RA), optou-se pela utilização da Nota do Consumidor ou da Média das Avaliações dos Consumidores (MA), ao invés de utilizar o índice de reputação consolidado.

Além do fato de que a técnica ANOVA necessita de uma variável métrica (Hair et al., 2005), essa escolha pode ser justificada pelos resultados obtidos por Utz, Kerkhof e Bos (2012), que indicaram que as avaliações de consumidores são consideradas mais importantes do que selos aferidos por sites, para indivíduos que buscam estabelecer uma confiança inicial, para realização de compras com uma empresa desconhecida. Na próxima seção, são apresentados os resultados obtidos junto à amostra.

\section{Resultados}

Uma vez definidos os escores (variáveis) que seriam utilizados no teste ANOVA, foram realizados testes para verificar se as suposições necessárias à realização do teste foram atendidas (Anderson et al., 2011). Foram identificados dois outliers na amostra, sendo um no grupo 1 (Prata+Bronze) e um no grupo 2 (Ouro). Essas duas ocorrências foram mantidas na amostra, uma vez que não foram identificadas justificativas para sua remoção. A Tabela 4 apresenta os testes de normalidade para os três grupos formados.

Tabela 4

Testes de Normalidade para as Amostras dos Grupos

\begin{tabular}{lllllll}
\hline \multirow{2}{*}{ Grupos } & \multicolumn{3}{l}{ Kolmogorov-Smirnov } & \multicolumn{3}{l}{ Shapiro-Wilk } \\
\cline { 2 - 7 } & Estatística & Gl & Sig. & Estatística & gl & Sig. \\
\hline Medalha 1 & .085 & 30 & .200 & .984 & 30 & .912 \\
Medalha 2 & .101 & 30 & .200 & .964 & 30 & .395 \\
Medalha 3 & .099 & 30 & .200 & .967 & 30 & .454 \\
\hline
\end{tabular}


Para fins de realização do teste, os dados foram categorizados da seguinte forma: Medalha 1.00 (Empresas com selo Prata ou Bronze), Medalha 2.00 (Empresas com selo Ouro), Medalha 3.00 (Empresas com selo Diamante).

Os resultados dos testes de normalidade indicam que, em nível de significância de $95 \%(\alpha=0,05)$, não há evidências de anormalidade nas amostras, atendendo, portanto, às suposições para realização do teste ANOVA (p-valor > 0,05).

Os erros encontrados nos grupos apresentaram um comportamento que pode ser caracterizado como independente. Além disso, não ficou evidente a existência de características de heterocedasticidade nos erros padronizados dos grupos, o que dá subsídios à realização do teste ANOVA.

A Tabela 5 apresenta as estatísticas descritivas relacionadas às características gerais dos grupos da amostra.

Tabela 5

Descrição dos Grupos da Amostra

\begin{tabular}{llll}
\hline Grupos & Contagem & Média & Variância \\
\hline Medalha 1 & 30 & 5,184 & 2,271452414 \\
Medalha 2 & 30 & 5,063 & 1,005614828 \\
Medalha 3 & 30 & 5,652333333 & 1,597287471 \\
\hline
\end{tabular}

Pôde-se perceber que as médias para os três grupos parecem muito próximas, o que gerou indícios de que não seriam encontradas evidências da diferença entre elas. Por fim, a Tabela 6 apresenta os resultados obtidos a partir do teste ANOVA.

Tabela 6

\section{Resultados do Teste ANOVA}

\begin{tabular}{lccccc}
\hline Fonte da Variação & SQ & gl & F & valor-p & F crítico \\
\hline Entre Grupos & 5,812908889 & 2 & 1,788824131 & 0,173249593 & 3,101295757 \\
Dentro dos Grupos & 141,3562867 & 87 & & & \\
Total & 147,1691956 & 89 & & & \\
\hline
\end{tabular}

Obteve-se o valor da estatística $\mathrm{F}$ de aproximadamente 1,79 , sendo inferior ao $\mathrm{F}$ crítico, de aproximadamente $3,1(\alpha=0,05)$. Além disso, o valor-p, de 0,17 , indica que não há evidências da existência de diferença das médias de reputação entre os grupos (lojas virtuais Diamante, Ouro e Prata + Bronze). Portanto, rejeita-se $\mathrm{H}_{\mathrm{A}}$ (Nem todas as médias são iguais) e mantém-se $\mathrm{H}_{0}$ (Todas as médias são iguais). A estatística $R^{2}$, obtida da razão entre SQ Dentro e SQ Total, indica que o fator confiança, representado pelos selos eBIT, explica aproximadamente $96 \%$ da variação do índice das médias das notas dos consumidores (MA).

\section{Análise dos Resultados}

O tamanho da amostra deve ser considerado um viés incorporado aos resultados desse estudo. No entanto, isso pode ser justificado pelo tempo de criação das bases de dados utilizadas, principalmente a 
base de dados do site Reclame Aqui. Verificou-se que muitas das empresas avaliadas pelo sistema eBIT, principalmente aquelas com selos Prata e Bronze, não possuem índices de avaliação no sistema do Reclame Aqui. A utilização dessa base de dados, composta de dados secundários, também pode apresentar vieses em sua coleta, afetando a mensuração dos construtos (Houston, 2004).

Por outro lado, considerando a relação teórica existente entre os construtos reputação e confiança, bem como as particularidades do mercado brasileiro, expressadas pelos escores utilizados, os resultados obtidos nesta pesquisa demonstram que existe incoerência quanto aos índices apresentados pelos sistemas de reputação, assim respondendo à questão de pesquisa.

O indicador $R^{2}>60 \%$ (95\% aproximadamente) demonstra que os selos de confiança do site eBIT são fatores relevantes para explicar a variação das médias das avaliações dos consumidores no sistema de reputação Reclame Aqui (Hair et al., 2005). Todavia, a obtenção do valor-p de 0,17, por meio do teste ANOVA, indicou que as médias de reputação (MA) das empresas com selos de confiança mais fortes se mostraram muito semelhantes às médias das empresas com selos de confiança mais fracos.

Do ponto de vista teórico, os achados reforçam a possibilidade de existência de outras variáveis que influenciam significativamente a relação entre os construtos confiança e reputação, no corpo teórico de sistemas de reputação.

De fato, outros estudos sobre os sistemas de reputação mencionam problemas relacionados a esta (por meio de propagandas, para minimizar os feedbacks negativos), a vendedores que compram e qualificam seus próprios produtos, a vendedores que estabelecem uma reputação inicial para aplicar golpes posteriores, à facilidade que os vendedores encontram para criar novos perfis para burlar feedbacks negativos, e ao fato de que os sistemas de reputação, em geral, são pagos pelas empresas avaliadas (Edelman, 2011; Saastamoinen, 2009).

Adicionalmente, a literatura indica que, apesar dos benefícios, os sistemas de reputação não solucionam totalmente o problema da assimetria de informação. Enquanto a mensuração dos feedbacks de clientes é realizada por meio de medidas quantitativas, o teor dos feedbacks é subjetivo e heterogêneo, uma vez que podem ser encontradas diferentes preferências entre os consumidores (Pavlou \& Dimoka, 2006).

Todavia, cabe o fomento de novos estudos, essencialmente nos campos de Sistemas de Informação e Marketing, para identificar as variáveis que afetam a relação investigada, levando em conta as particularidades do mercado e as dos sistemas de reputação brasileiros.

Do ponto de vista empírico, os resultados trazem implicações para consumidores, varejistas, órgãos regulatórios, empresas mantenedoras de sistemas de reputação e demais interessados na composição e na divulgação dos escores de reputação e confiança.

Do ponto de vista da aplicação do método, até onde se investigou pelos autores do presente trabalho, nenhuma pesquisa havia utilizado a abordagem de comparação de médias por meio de teste ANOVA para avaliação de coerência de sistemas de reputação.

\section{Conclusões e Pesquisas Futuras}

Esta pesquisa verificou a relação teórica entre os construtos confiança e reputação, quando aplicados na análise de sistemas de reputação. Além disso, foi apresentada uma nova possibilidade em termos de abordagem para análise de coerência de escores de reputação, utilizando o teste ANOVA para comparação de médias. Atendendo ao principal propósito desse trabalho, as análises indicaram que não há coerência entre os escores testados no estudo, uma vez que estes não conseguem atender as relações teóricas pressupostas na literatura. 
Desta forma, os resultados da pesquisa sugerem a existência de outras variáveis presentes na relação teórica entre os construtos confiança e reputação, no contexto verificado. Espera-se que as relações, as práticas e o papel desses mecanismos de governança sejam estudados para melhor compreensão do comércio eletrônico no âmbito nacional.

Neste sentido, as pesquisas futuras devem investigar quais as fontes de vieses e características dos escores dos sistemas de reputação brasileiros, possibilitando o aprofundamento do modelo teórico que envolve os construtos confiança e reputação.

Do ponto de vista metodológico, há a expectativa de que as bases utilizadas neste trabalho se tornem maiores no decorrer dos próximos anos, possibilitando a aplicação de outras técnicas estatísticas, como o teste qui-quadrado. Há, ainda, a necessidade de estudar a composição das medidas agregadas, que foram analisadas neste estudo, considerando a possibilidade de expandi-las ou substituí-las por medidas mais apropriadas aos construtos confiança e reputação. Possibilidades alternativas de pesquisa nesta área envolvem o desenvolvimento de métricas e técnicas para avaliação de escores e selos aferidos pelos sistemas de reputação nacionais. Outras pesquisas são necessárias no sentido de identificar se há divergências entre os índices de empresas de diferentes segmentos do mercado.

O presente estudo ainda traz achados de cunho empírico. A partir dos resultados, infere-se que o consumidor deve buscar informações do maior número de fontes possível, para que os riscos inerentes ao processo de compra sejam reduzidos. No que concerne às empresas e desenvolvedores de sistemas de reputação, sugere-se a revisão de escores utilizados para que estes sejam aderentes aos conceitos que se propõem a medir (confiança e reputação). Uma possível alternativa é a integração dos diferentes mecanismos de reputação, com a possibilidade de geração de um escore composto, garantindo a coerência entre mecanismos. Outra sugestão, envolvendo os órgãos de defesa do consumidor, está relacionada ao investimento em sistemas ou empresas de certificação, no sentido de garantir a integridade dos índices divulgados acerca de empresas de comércio eletrônico.

\section{Notas}

\footnotetext{
${ }^{1}$ Após as últimas atualizações, a lista completa com as empresas mais queridas do sistema eBIT em 2011 foi removida e não está mais disponível para consulta.

${ }^{2}$ Quando os dados foram coletados, o tempo de cadastro para cálculo do índice era de 30 dias.
}

\section{Referências}

Albertin, A. L., \& Moura, R. M. (1998). Comércio eletrônico: seus aspectos de segurança e privacidade. Revista de Administração de Empresas, 38(2), 49-61. doi: 10.1590/S0034-75901998000200006

Amblee, N., \& Bui, T. (2008). Can brand reputation improve the odds of being reviewed on-line. International Journal of Electronic Commerce, 12(3), 11-28. Recuperado de http://www.tandfonline.com/doi/pdf/10.2753/JEC1086-4415120302\#.VgmQ55cR40k. doi: 10.2753/JEC1086-4415120302

Anderson, D. R., Sweeney, D. J., \& Williams, T. A. (2011). Estatística aplicada à administração e economia (2a ed.). São Paulo: Cengage Learning.

Azderska, T., \& Jerman-Blazic, B. (2013). A holistic approach for designing human-centric trust systems. Systemic Practice and Action Research, 26(5), 417-450. doi: 10.1007/s11213-012-9259-3 
Ba, S., \& Pavlou, P. A. (2002). Evidence of the effect of trust building technology in electronic markets: price premiums and buyer behavior. MIS Quarterly, 26(3), 243-268. doi: 10.2307/4132332

Bae, S., \& Lee, T. (2011). Gender differences in consumers perception of online consumer reviews. Electronic Commerce Research, 11(2), 201-214. doi: 10.1007/s10660-010-9072-y

Ballester, E. D., \& Espallardo, M. H. (2008). Effect of brand associations on consumer reactions to unknown on-line brands. International Journal of Electronic Commerce, 12(3), 81-113. doi: 10.2753/JEC1086-4415120305

Benedicktus, R. L., Brady, M. K., Darke, P. R., \& Voorhees, C. M. (2010). Conveying trustworthiness to online consumers: reactions to consensus, physical store presence, brand family, and generalized suspicion. Journal of Retailing, 86(4), 322-335. doi: 10.1016/j.jretai.2010.04.002

Benlian, A., \& Hess, T. (2011). The signaling role of IT features in influencing trust and participation in online communities. International Journal of Electronic Commerce, 15(4), 7-56. doi: 10.2753/JEC1086-4415150401

Black, T. (1999). Doing quantitative research in the social sciences: an integrated approach to research design, measurement and statistics. London: Sage.

Butler, J. K. (1991). Toward understanding and measuring conditions of trust: evolutions of a condition of trust inventory. Journal of Management, 17(3), 643-663. doi: 10.1177/014920639101700307

Casado, A. M., \& Pelaez, J. I. (2014). Intangible management monitors and tools: reviews. Expert Systems with Applications, 41(4), 1509-1529. doi: 10.1016/j.eswa.2013.08.048

Casaló, L. V., Flavián, C., \& Guinalíu, M. (2011). The generation of trust in the online services and product distribution: the case of Spanish electronic commerce. Journal of Electronic Commerce Research, 12(3), 199-213.

Cernev, A. K., \& Leite, J. A. (2005, setembro). Segurança na internet: a percepção dos usuários como fator de restrição ao comércio eletrônico no Brasil. Anais do Encontro Nacional da Associação Nacional de Pós-Graduação e Pesquisa em Administração, Brasília, DF, Brasil, 29.

Chiu, C.-M., Huang, H.-Y., \& Yen, C.-H. (2010). Antecedents of trust in online auctions. Electronic Commerce Research and Applications, 9(2), 148-159. doi: 10.1016/j.elerap.2009.04.003

Cho, S.-E. (2010). Perceived risks and customer needs of geographical accessibility in electronic commerce. Electronic Commerce Research and Applications, 9(6), 495-506. doi: 10.1016/j.elerap.2010.02.005

Corbitt, B. J., Thanasankit, T., \& Yi, H. (2003). Trust and e-commerce: a study of customer perceptions. Electronic Commerce Research and Applications, 2(3), 203-215. Recuperado de http://ac.elscdn.com/S1567422303000243/1-s2.0-S1567422303000243-main.pdf?_tid=a5268258-661611e5-9ac6-00000aab0f6c\&acdnat=1443468495_a08988bed1a12f0728b07aa0b36787ab. doi: $10.1016 /$ S1567-4223(03)00024-3

Ebit A Certificação dos Consumidores. (n.d.a). Conheça as medalhas E-bit. Recuperado de http://www.ebit.com.br/medalhas-e-bit

Ebit A Certificação dos Consumidores. (n.d.b). Reputação das lojas. Recuperado de http://www.ebit.com.br/avaliacao-lojas

Ebit Empresa. (n.d.). Troféu diamante: comércio eletrônico conhece as melhores lojas do setor. Recuperado de https://empresa.ebit.com.br/imprensa_trofeu_2011.asp 
Edelman, B. (2011). Adverse selection in online "trust" certifications and search results. Electronic Commerce Research and Applications, 10(1), 17-25. Recuperado de http://ac.elscdn.com/S1567422310000487/1-s2.0-S1567422310000487-main.pdf?_tid=2e95be64-661711e5-be80-00000aab0f26\&acdnat=1443468726_2bf01f1b2aaf4910955f8e72ab3e4e21. doi: 10.1016/j.elerap.2010.06.001

Eid, M. I. (2011). Determinants of e-commerce customer satisfaction, trust, and loyalty in Saudi Arabia. Journal of Electronic Commerce Research, 12(1), 78-93.

Fuller, M. A., Serva, M. A., \& Benamati, J. S. (2007). Seeing is believing: the transitory influence of reputation information on e-commerce trust and decision making. Decision Sciences, 38(4), 675699. doi: 10.1111/j.1540-5915.2007.00174.x

Fundação de Proteção e Defesa do Consumidor. (2013). Cadastro de reclamações fundamentadas 2012. Recuperado de http://www.procon.sp.gov.br/pdf/acs_ranking_2012.pdf

Gregg, D. G., \& Walczak, S. (2010). The relationship between website quality, trust and price premiums at online auctions. Electronic Commerce Research, 10(1), 1-25. Retrieved from http://download.springer.com/static/pdf/106/art\%253A10.1007\%252Fs10660-010-9044-

2.pdf?originUrl=http $\% 3 \mathrm{~A} \% 2 \mathrm{~F} \% 2 \mathrm{Flink}$. springer.com\%2Farticle $\% 2 \mathrm{~F} 10.1007 \% 2 \mathrm{Fs} 10660-010-9044-$ $2 \&$ token $2=\exp =1443470200$ acl $=\% 2 \mathrm{Fstatic} \% 2 \mathrm{Fpdf} \% 2 \mathrm{~F} 106 \% 2 \mathrm{Fart} \% 25253 \mathrm{~A} 10.1007 \% 25252 \mathrm{Fs} 10$ 660-010-9044-

2.pdf\%3ForiginUr1\%3Dhttp\%253A\%252F\%252Flink.springer.com\%252Farticle\%252F10.1007\%2 52Fs 10660-010-9044-

$2 * \sim \mathrm{hmac}=\mathrm{c} 6755 \mathrm{e} 90 \mathrm{c} 78 \mathrm{acc} 93 \mathrm{f} 64669018757 \mathrm{f} 20918981918 \mathrm{cf} 1402011 \mathrm{f} 45525680 \mathrm{cbb} 687 . \quad$ doi: $10.1007 / \mathrm{s} 10660-010-9044-2$

Guay, D., \& Ettwein, J. (1998). Internet commerce basics. Electronic Markets, 8(1), 12-15. doi: $10.1080 / 10196789800000004$

Hair, J. F., Jr., Babin, B., Money, A. H., \& Samouel, P. (2005). Fundamentos de métodos de pesquisa em administração. Porto Alegre: Bookman.

Hartono, E., Holsapple, C. W., Kim, K. Y., Na, K. S., \& Simpson, J. T. (2014). Measuring perceived security in B2C electronic commerce website usage: a respecification and validation. Decision Support Systems, 62, 11-21. doi: 10.1016/j.dss.2014.02.006.

Houston, M. B. (2004). Assessing the validity of secondary data proxies for marketing constructs. Journal of Business Research, 57(2), 154-161. doi: 10.1016/s0148-2963(01)00299-5

Josang, A., Ismail, R., \& Boyd, C. (2007). A survey of trust and reputation systems for online service provision. Decision Support Systems, 43(2), 618-644. doi: 10.1016/j.dss.2005.05.019

Karahanna, E., Williams, C. K., Polites, G. L., Liu, B., \& Seligman, L. (2013). Uncertainty avoidance and consumer perceptions of global e-commerce sites: a multi-level approach. Drake Management Review, 3(1), 12-47.

Kim, C., Tao, W., Shin, N., \& Kim, K.-S. (2010). An empirical study of customers' perceptions of security and trust in-payment systems. Electronic Commerce Research and Applications, 9(1), 84-95. Recuperado de http://ac.els-cdn.com/S1567422309000283/1-s2.0-S1567422309000283main.pdf?_tid=5538e4a8-661a-11e5-9a51-

00000aacb35e\&acdnat=1443470079_f5b0d7b508313018c82b012d966167ac. doi: 10.1016/j.elerap.2009.04.014

Kim, D. J., Ferrin, D. L., \& Rao, H. R. (2008). A trust-based consumer decision-making model in electronic commerce: the role of trust, perceived risk, and their antecedents. Decision Support Systems, 44(2), 544-564. doi: 10.1016/j.dss.2007.07.001 
Kim, H.-W., \& Xu, Y. (2007). Drivers of price premium in e-markets. Communications of the ACM, 50(11), 91-95. doi: 10.1145/1297797.1297803

Kim, H.-W., Xu, Y., \& Gupta, S. (2012). Which is more important in internet shopping, perceived price or trust. Electronic Commerce Research and Applications, 11(3), 241-252. Recuperado de http://ac.els-cdn.com/S1567422311000342/1-s2.0-S1567422311000342-main.pdf?_tid=83097348661a-11e5-aa9d-00000aab0f6c\&acdnat=1443470155_f812f3bd8a8d0868fde269dc6606d557. doi: 10.1016/j.elerap.2011.06.003

Kollock, P. (1999). The production of trust in online markets. In E. Lawler, S. Thyne, \& H. Walker (Eds.), Advances in group processes (Vol. 16, pp. 99-123). Greenwich, CT: JAI Press. Recuperado de http://www.connectedaction.net/wp-content/uploads/2009/05/1999-peterkollock-the-production-of-trust-in-online-markets.htm

Kovacs, M.-H., \& Farias, S. A. (2004). Dimensões de riscos percebidos nas compras pela internet. RAEeletrônica, $3(2), \quad 1-18 . \quad$ Recuperado de http://rae.fgv.br/sites/rae.fgv.br/files/artigos/10.1590_S1676-56482004000200004.pdf.

Lewicki, R. J., \& Bunker, B. B. (1995). Trust in relationships: a model of trust development and decline. In B. B. Bunker \& J. Z. Rubin (Eds.), Conflict, cooperation and justice (pp. 133-173). San Francisco, CA: Jossey-Bass. Recuperado de http://www.researchgate.net/publication/232534885_Trust_in_relationships_A_model_of_devel opment_and_decline

Liao, C., Liu, C.-C., \& Chen, K. (2011). Examining the impact of privacy, trust and risk perceptions beyond monetary transactions: an integrated model. Electronic Commerce Research and Applications, 10(6), 702-715. Recuperado de http://ac.els-cdn.com/S1567422311000408/1-s2.0S1567422311000408-main.pdf?_tid=0764502a-661d-11e5-a0e4-

00000aacb362\&acdnat=1443471236_50d68b1d3dc09b6c82e78a082fa42a4d. doi: 10.1016/j.elerap.2011.07.003

Lin, Z., Li, D., Jamananchi, B., \& Huang, W. (2006). Reputation distribution and consumer-to-consumer online auction market structure: an exploratory study. Decision Support Systems, 41(2), 435-448. doi: $10.1016 /$ j.dss.2004.07.006

McKnight, D. H., Choudhury, V., \& Kacmar, C. (2002). Developing and validating trust measures for e-commerce: an integrative typology. Information Systems Research, 13(3), 334-359. doi: 10.1287/isre.13.3.334.81

McKnight, D. H., Cummings, L. L., \& Chervany, N. L. (1998). Initial trust formation in new organizational relationships. Academy of Management Review, 23(3), 473-490. doi: 10.5465/AMR.1998.926622

Melnik, M. I., \& Alm, J. (2002). Does a seller e-commerce reputation matter? Evidence from eBay auctions. The Journal of Industrial Economics, 50(3), 337-349. doi: 10.1111/1467-6451.00180

Meyerson, D., Weick, K. E., \& Kramer, R. M. (1996). Swift trust and temporary groups. In R. M. Kramer \& T. R. Tyler (Eds.), Trust in organizations: frontiers of theory and research (pp. 166195). Thousand Oaks, CA: Sage. Recuperado de http://books.google.com.br/books?id=A_8LbcsgrNMC\&pg=PA189\&dq=swift+trust+and+temporar $\mathrm{y}+$ groups\&hl=pt-PT\&sa=X\&ei=z8CmULGxMIWO8wS90oDQDw\&ved=0CDMQ6AEwAQ

Mudambi, S. M., \& Schuff, D. (2010). What makes a helpful online review? A study of customer reviews on Amazon.com. MIS Quarterly, 34(1), 185-200. 
Park, I., Bhatnagar, A., \& Rao, H. R. (2010). Assurance seals, on-line customer satisfaction, and repurchase intention. International Journal of Electronic Commerce, 14(3), 11-34. doi: 10.2753/JEC1086-4415140302

Pavlou, P. A., \& Dimoka, A. (2006). The nature and role of feedback text comments in online marketplaces: implications for trust building, price premium and seller diferentiation. Information Systems Research, 17(4), 392-414. doi: 10.1287/isre.1060.0106

Pavlou, P. A., Liang, H. G., \& Xue, Y. J. (2007). Understanding and mitigating uncertainty in online exchange relationships: a principal-agent perspective. MIS Quarterly, 31(1), 105-136.

Pizzutti, C., \& Fernandes, D. (2010). Effect of recovery efforts on consumer trust and loyalty in e-tail: a contingency model. International Journal of Electronic Commerce, 14(4), 127-160. Recuperado de http://www.tandfonline.com/doi/pdf/10.2753/JEC1086-4415140405\#.Vgmf8flViko. doi: 10.2753/JEC1086-4415140405

ReclameAqui. (n.d.a). Como funcionam os critérios para a avaliação das empresas? Recuperado de http://www.reclameaqui.com.br/como_funciona/como-funcionam-os-criterios-para-a-avaliacaodas-empresas_19/

ReclameAqui. (n.d.b). Rankings das empresas mais reclamadas por categoria. Recuperado de http://www.reclameaqui.com.br/

Rempel, J. K., Holmes, J. G., \& Zanna, M. P. (1985). Trust in close relationships. Journal of Personality and Social Psychology, 49(1), 95-112. doi: 10.1037/0022-3514.49.1.95

Resnick, P., Zeckhauser, R., Friedman, E., \& Kuwabara, K. (2000). Reputation systems. Communications of the Acm, 43(12), 45-48. doi: 10.1145/355112.355122

Rotter, J. B. (1971). Generalized expectancies for interpersonal trust. American Psychologist, 26(5), 443-452. doi: 10.1037/h0031464

Saastamoinen, J. (2009). Returns on reputation in retail e-commerce. Journal of Electronic Commerce Research, 10(4), 196-219. Recuperado de http://ojs.jecr.org/jecr/sites/default/files/10_4_p01.pdf

Shapiro, D. L., Sheppard, B. H., \& Cheraskin, L. (1992). Business on a handshake. Negotiation Journal, 8(4), 365-377. doi: 10.1111/j.1571-9979.1992.tb00679.x

Smith, R., \& Shao, J. (2007). Privacy and e-commerce: a consumer-centric perspective. Electronic Commerce Research, 7(2), 89-116. Recuperado de http://download.springer.com/static/pdf/849/art\%253A10.1007\%252Fs10660-007-9002-

9.pdf?originUrl=http $\% 3 \mathrm{~A} \% 2 \mathrm{~F} \% 2 \mathrm{Flink}$. springer.com\%2Farticle $\% 2 \mathrm{~F} 10.1007 \% 2 \mathrm{Fs} 10660-007-9002-$ $9 \&$ token $2=\exp =1443472898 \sim$ acl $=\% 2 \mathrm{Fstatic} \% 2 \mathrm{Fpdf} \% 2 \mathrm{~F} 849 \% 2 \mathrm{Fart} \% 25253 \mathrm{~A} 10.1007 \% 25252 \mathrm{Fs} 10$ 660-007-9002-

9.pdf\%3ForiginUr1\%3Dhttp $\% 253 \mathrm{~A} \% 252 \mathrm{~F} \% 252 \mathrm{Flink}$. springer.com\%252Farticle\%252F10.1007\%2 52Fs10660-007-9002-

$9^{*} \sim \mathrm{hmac}=\mathrm{dc} 3605 \mathrm{eec} 5 \mathrm{e} 214 \mathrm{a} 7 \mathrm{e} 653524 \mathrm{f} 2 \mathrm{~d} 5 \mathrm{ed} 350929 \mathrm{~d} 349 \mathrm{daaf} 110991 \mathrm{fc} 33 \mathrm{f} 1 \mathrm{~b} 5 \mathrm{~b} 5 \mathrm{a} 196 \mathrm{e} \quad$ doi: $10.1007 / \mathrm{s} 10660-007-9002-9$

Standifird, S. S. (2001). Reputation and e-commerce: eBay auctions and the asymmetrical impact of positive and negative ratings. Journal of Management, 27(3), 279-295. doi: $10.1177 / 014920630102700304$

Standifird, S. S., Weinstein, M., \& Neyer, A. D. (1999, August). Establishing reputation on the Warsaw stock exchange: international brokers as legitimating agents. Proceedings of Academy of Management, Chicago, IL, USA, 59. 
Swamynathan, G., Almeroth, K. C., \& Zhao, B. Y. (2010). The design of a reliable reputation system. Electronic Commerce Research, 10(3), 239-270. doi: 10.1007/s10660-010-9064-y

Turban, E., \& King, D. (2004). Comércio eletrônico: estratégia e gestão. Prentice Hall: São Paulo.

Utz, S., Kerkhof, P., \& Bos, J. van den (2012). Consumers rule: how consumer reviews influence trustworthiness of online stores. Electronic Commerce Research and Applications, 11(1), 49-58. doi: 10.1016/j.elerap.2011.07.010

Utz, S., Matzat, U., \& Snijders, C. (2009). On-line reputation systems: the effects of feedback comments and reactions on building and rebuilding trust in on-line auctions. International Journal of Electronic Commerce, 13(3), 95-118. doi: 10.2753/JEC1086-4415130304

Vavilis, S., Petkovic, M., \& Zannone, N. (2014). A reference model for reputation systems. Decision Support Systems, 61(1), 147-154. doi: 10.1016/j.dss.2014.02.002

Vieira, V. A. (2008). Um modelo dos antecedentes da lealdade no varejo eletrônico [Edição Especial]. Revista de Administração Contemporânea, 12, 65-87. doi: 10.1590/S1415-65552008000500004

Vieira, V. A. (2010). Mensuração da qualidade de serviço no varejo eletrônico e seu impacto sobre as intenções comportamentais. Revista de Administração de Empresas, 50(2), 199-214. doi 10.1590/S0034-75902010000200006

Wartick, S. L. (2002). Measuring corporate reputation definition and data. Business and Society, 41(4), 371-392. doi: 10.1177/0007650302238774

Whitmeyer, J. M. (2000). Effects of positive reputation systems. Social Science Research, 29(2), 188207. doi: $10.1006 /$ ssre. 1999.0663

Wu, C. S., Cheng, F. F., \& Yen, D. C. (2014). The influence of seller, auctioneer, and bidder factors on trust in online auctions. Journal of Organizational Computing and Electronic Commerce, 24(1), 36-57. doi: 10.1080/10919392.2014.866502

Yoo, B., \& Kim, K. (2012). Does popularity decide rankings or do rankings decide popularity? An investigation of ranking mechanism design. Electronic Commerce Research and Applications, 11(2), 180-191. doi: 10.1016/j.elerap.2011.12.007

You, W., Liu, L., Xia, M., \& Lv, C. (2011). Reputation inflation detection in a Chinese C2C market. Electronic Commerce Research and Applications, 10(5), 510-519. doi: 10.1016/j.elerap.2011.06.001

Zhang, S. Y., Chen, Y. W., \& Wang, E. P. (2004). Consumers' risk perception in B2C electronic commerce. International Journal of Psychology, 39(5/6), 342-342.

Zhou, R. F., \& Hwang, K. (2007). PowerTrust: a robust and scalable reputation system for trusted peerto-peer computing. IEEE Transactions on Parallel and Distributed Systems, 18(4), 460-473. doi: $10.1109 /$ tpds.2007.1015

\title{
Dados dos Autores
}

\author{
Douglas de Lima Feitosa \\ Av. 9 de Julho, 2029, Bela Vista, 01313-902, São Paulo, SP, Brasil. E-mail: douglas-feitosa@uol.com.br \\ Leandro Sumida Garcia \\ Av. dos Estados, 5001, ARI, 09210-580, Santo André, SP, Brasil. E-mail: leandro.sumida@gmail.com
}

